\title{
EFFECTS OF INFERIOR TEMPORAL LESIONS ON DISCRIMINATION OF STIMULI DIFFERING IN ORIENTATION ${ }^{1}$
}

\author{
EDWARD J. HOLMES ${ }^{2}$ AND CHARLES G. GROSS \\ Department of Psychology, Princeton University, Princeton, New Jersey 08540
}

Received March 21, 1984; Revised June 4, 1984; Accepted June 6, 1984

\begin{abstract}
Monkeys with inferior temporal (IT) cortex lesions were compared with normal and operated control monkeys on a series of two-choice visual discrimination problems. In some problems, the discriminanda were different patterns or objects whereas, in other problems, the discriminanda were identical patterns or objects presented in different orientations. The animals with IT lesions were significantly impaired in learning to discriminate the different patterns and objects; however, they were not impaired in learning to discriminate stimuli which differed only in orientation by $60^{\circ}$ or more. These results help to specify further the role of the IT cortex in visual associative learning by demonstrating that there are some types of visual discriminations which are not sensitive to IT lesions.
\end{abstract}

Bilateral ablation of the inferior temporal (IT) cortex in monkeys produces an impairment in visual learning while leaving basic visual thresholds, such as for visual acuity, unchanged (see reviews by Gross, 1973; Dean, 1976). The severity of impairment in visual discrimination learning following IT lesions depends on the difficulty of the discrimination task as measured by the number of trials normal monkeys require to learn it. The harder the task for normal monkeys, the greater the deficit for animals with IT lesions. Recently, however, we discovered a type of visual discrimination that animals with IT lesions acquire as readily as normal animals, namely, tasks in which the discriminanda are two identical patterns differing in orientation by either $180^{\circ}$, e.g., A versus $\mathbf{V}$, or $90^{\circ}$, e.g., A versus $>$ (Gross, 1978). This sparing of impairment on orientation discriminations occurred even on tasks the normal animals found relatively difficult. By contrast the animals with IT lesions were impaired in discriminating stimuli differing in orientation if the stimuli were line segments (e.g., I versus -) rather than patterns.

The purpose of the present study was to analyze further this intriguing phenomenon of an absence of impairment on orientation discriminations by monkeys with IT lesions. First, we wanted to know whether the sparing of impairment also held for the discrimination of orientation differences other than 90 or $180^{\circ}$. Second, we wanted to determine whether the sparing of impairment would occur with three-dimensional objects as well as two-dimensional patterns. Third, we wanted to reinvestigate the presence of impairment when the discriminanda are rotated line segments.

${ }^{1}$ This research was supported by Grants BNS 75-25634 and 79 05589 from the National Science Foundation, by Grant MH 19420 from the National Institutes of Health, and by the Spenser Foundation.

${ }^{2}$ To whom correspondence should be addressed, at his present address: Department of Neuruscience, Rose Kennedy Center, Einstein College of Medicine, 1410 Pelham Parkway, Bronx, NY 10461.

\section{Materials and Methods \\ Subjects and surgery}

The subjects were 13 experimentally naive cynomolgus monkeys (Macaca fascicularis) weighing between 2.3 and $3.5 \mathrm{~kg}$ during initial training. Five monkeys were given bilateral lesions of IT cortex (IT group), three were given bilateral lesions of lateral striate cortex and served as the operated control group (S group), and five remained unoperated (U group). The $S$ and $U$ groups will be referred to collectively as the control groups.

The lesions of the IT cortex (i.e., cytoarchitectonic area TE of von Bonin and Bailey, 1947) were intended to begin $10 \mathrm{~mm}$ anterior to and parallel with the ascending portion of the inferior occipital sulcus. The lesion was to extend for $10 \mathrm{~mm}$ anteriorly, bounded dorsally by the fundus of the superior temporal sulcus and ventrally by the medial bank of the occipitotemporal sulcus.

The lesions of lateral (macular) striate cortex were intended to begin 2 to $3 \mathrm{~mm}$ posterior to the lunate sulcus and to extend caudally and parallel to the sagittal fissure for approximately $15 \mathrm{~mm}$. The dorsal limit of the lesion was intended to begin $5 \mathrm{~mm}$ from the sagittal fissure and to extend ventrally and parallel with the lunate sulcus for approximately $20 \mathrm{~mm}$. The lesion was intended to remove the representation of approximately the central $6^{\circ}$ of the visual field. The surgical methods for both lesions have been described previously (Gross, 1978).

\section{Histology}

The methods of perfusing, sectioning, staining, and reconstructing the lesions have been described elsewhere (Cowey and Gross, 1970). Reconstructions of the extent of the IT lesions are shown in Figure 1. Both the IT and the lateral striate lesions were approximately as intended.

\section{Behavioral apparatus and procedures}

Pattern discrimination. Pattern discrimination training was carried out in a sound-insulated chamber. Two translucent Gerbrands keys, $3.2 \mathrm{~cm}$ in diameter and $30.7 \mathrm{~cm}$ apart (center to center), were mounted on one wall of the chamber. The discriminanda were projected onto the keys by rear projection readout units (series 10, Industrial Electronics Engineers, Inc.). An automatic liquid dispenser was mounted on the same wall $25.6 \mathrm{~cm}$ below the level of the keys. The interior of 


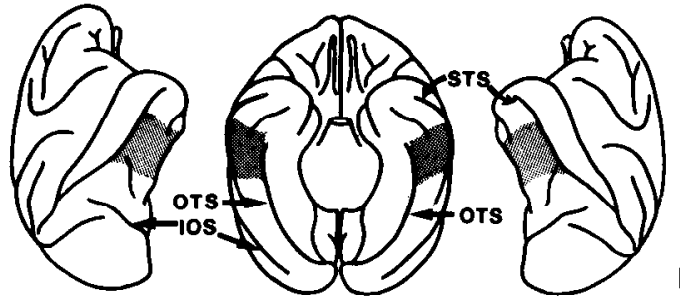

IT 1
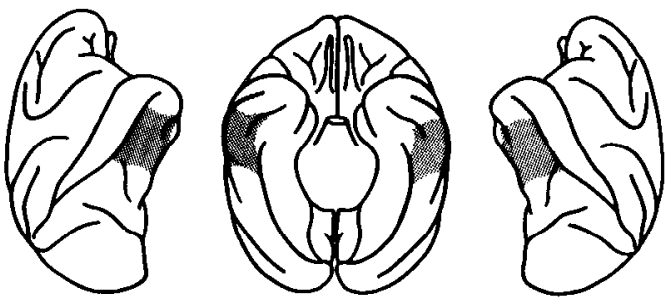

IT.2
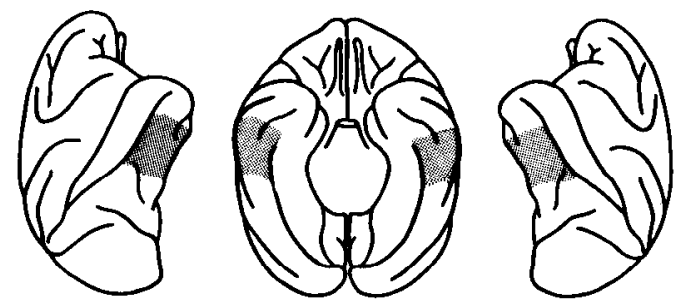

IT.3
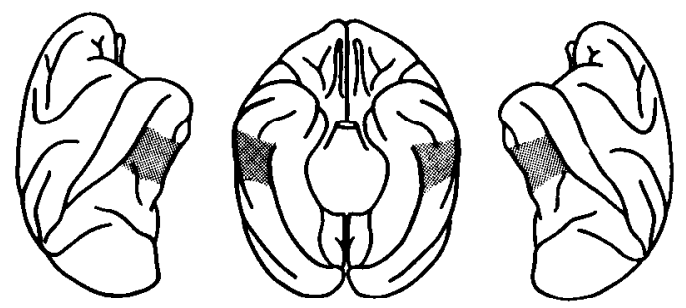

IT. 4
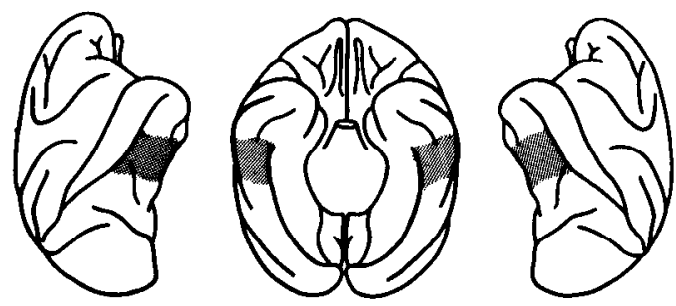

$1 T \cdot 5$

Figure 1. Reconstructions of the extent of the IT lesions. The stippled area indicates the region of damage. IOS, inferior occipital sulcus; OTS, occipitotemporal sulcus; STS, superior temporal sulcus.

the chamber was illuminated from above and a masking noise was present during training. The left-right spatial position of the stimuli was determined automatically, according to a balanced pseudo-random order.

The monkeys were trained on the pattern discrimination tasks for 100 trials/day, 7 days each week, while on a water deprivation schedule. The intertrial interval during postoperative training was $5.9 \mathrm{sec}$ and any response (i.e., key press) during the intertrial interval would restart the interval. A response to either or both keys turned the stimulus projectors off and ended the trial. Simultaneous presses of both keys counted as an error. A response to the rewarded stimulus was followed by the presentation of $0.25 \mathrm{ml}$ of orange juice.

Prior to the start of discrimination training there were three phases of pretraining. In the first phase, both response keys were illuminated with a white square and the animals were trained to press either one of the illuminated keys for a reward. The intertrial interval was $3.5 \mathrm{sec}$ and the animals were allowed to respond for 100 trials. In the second phase, the animals were trained to discriminate the key illuminated with a white square from a dark key. Responses to the illuminated key were rewarded as in the first phase of training. Criterion performance on this task (and all subsequent tasks in the chamber) was 90 correct responses in a 100-trial session. The final phase of pretraining was the same as the second phase except that the intertrial interval was increased to $5.9 \mathrm{sec}$

Object discrimination. Object discrimination training was carried out in a Wisconsin General Test Apparatus (WGTA). In the WGTA, an opaque screen separated an illuminated compartment containing the animal's cage from an illuminated compartment containing a form board. The form board contained two food wells which were $35.5 \mathrm{~cm}$ apart (center to center) and located $20 \mathrm{~cm}$ from the animal's cage. A one-way screen allowed the experimenter to observe the subject's response when the opaque screen was raised. Training was conducted in a darkened room with masking noise.

The animals were trained on the object discriminations for 50 trials/ day, 7 days each week, while partially food deprived. On each training trial, the food wells were covered by the discriminanda which were mounted on black $10.5 \times 9.0 \mathrm{~cm}$ plaques. The discriminanda could be displaced only in the anterior-posterior direction. Reward for displacing the correct stimulus was a raisin in the food well. The left-right spatial position of the stimuli was balanced according to a predetermined, pseudo-random schedule. On all problems the animals were trained to a criterion of 45 correct responses in any 50 consecutive trials.

To avoid the animals' use of extraneous cues such as dirt or scratches on the stimuli, two sets of stimuli were made for each discrimination problem, and the members of each set were randomly interchanged during training. For some of the discriminanda, the orientation of the stimuli was changed between the rewarded and unrewarded positions at the end of each training day.

Preoperative learning and postoperative retention. All subjects were initially trained in the automatic test chamber. After pretraining was completed, the subjects were trained to discriminate the patterns (shown in Fig. 2) until the criterion was reached. The subjects were assigned to one of the three groups in a fashion that equated the performance of the groups on this task as equally as possible. Within 2 weeks after reaching the learning criterion, the subjects received their designated surgical treatment.

Approximately 4 weeks after reaching the original criterion, the IT and $\mathrm{U}$ groups were retrained on the discriminations. The animals with lateral striate lesions were retrained after an additional 4 weeks to allow their residual vision to approach an asymptotic level.

Postoperative learning. Following the completion of the postoperative retention task, the subjects were trained on a series of 30 two-choice visual discrimination tasks. Two of the unoperated animals and two of the animals with IT lesions (IT-2 and IT-4) were trained only on the first 10 discrimination problems. The first task was designated problem 1 , the second problem 2, and so on. Problems 1 to 10 and 23 to 30 were two-dimensional discriminations either of patterns or of line segments and were trained in the automatic test chamber. Problems 11 to 22 were three-dimensional object discriminations and were trained in the WGTA. The discriminanda in problems 4,10 , and 24 were the pairs of different patterns shown in Figure 3. Problems 5, 6, 8, and 26 involved the discrimination of identical patterns differing in orientation by $180^{\circ}$ (Fig. 4, top). Problems 2, 3, and 7 involved identical patterns differing in orientation by $90^{\circ}$ (Fig. 4, middle). Problems 27 and 30 involved identical patterns differing in orientation by $45^{\circ}$ (Fig. 4 , bottom left). Problems 23, 25, and 29 involved identical patterns differing in orientation by $30^{\circ}$ (Fig. 4, bottom right). Problems 1, 9, and 28 involved identical line segments differing in orientation by either 180 or $90^{\circ}$ (Fig. 6)

The three-dimensional discriminanda were small colored toys such as cars, animals, and airplanes. Problems 11, 12, and 13 involved pairs of different objects. Problems 14, 17, and 19 involved pairs of identical objects differing in orientation by $120^{\circ}$. Problems 15,18 , and 21 involved pairs of identical objects differing in orientation by $60^{\circ}$. Problems 16, 20, and 22 involved pairs of identical objects differing in orientation by $30^{\circ}$.

Statistical analysis. On each individual problem a one-tailed MannWhitney $U$ test was used to assess the significance of the differences between the groups. Data were not pooled across problems.

\section{Results}

\section{Postoperative retention}

Postoperative retention of the discriminations learned prior to surgery was assessed by the savings measure

preoperative errors - postoperative errors preoperative errors + postoperative errors 
and is summarized in Figure 2. The $\mathrm{S}$ group showed only slightly poorer savings than the $\mathrm{U}$ group, but the difference was significant ( $U=0, p=0.018$ ). As expected, the IT group was very severely impaired, with four of the five animals showing negative savings. The IT group was significantly impaired relative to both the $U(U=0, p=0.004)$ and the $\mathrm{S}$ groups ( $U$ $=0, p=0.018$ ).

\section{Postoperative acquisition}

Different patterns (Fig. 3). On all three problems (problems 4,10 , and 24) involving different patterns as discriminanda, every I'T animal made more errors than did every control animal. The difference between the IT and $U$ groups was significant at the 0.05 level for each problem $(U=0)$, as was the difference between the IT and S groups. Furthermore, the severity of the IT deficit remained similar across the approximately 18 months between problem 4 and problem 24 . The performance of the two control groups was very similar on all three problems $(U \geq 3)$.

Rotated patterns (Fig. 4). On all problems in which the discriminanda differed only in their orientation by $180^{\circ}$ (problems $5,6,8$, and 26 ) or by $90^{\circ}$ (problems 2,3 , and 7 ), the performance of the IT group was indistinguishable from that of both control groups $(U \geq 3)$. The performance of the two control groups was also very similar $(U \geq 4)$ on all of these problems with the exception of the first, problem 2. On that task, the $\mathrm{S}$ group was significantly poorer than the $\mathrm{U}$ group ( $U$ $=1, p=0.036$ ).

By contrast, on both problems in which the discriminanda differed by $45^{\circ}$ and on all three problems in which they differed by $30^{\circ}$, every IT animal made many more errors than did every control animal; the group differences were significant at the 0.05 level in all cases. The performance of the two control groups was indistinguishable on all five problems $(U \geq 4)$. Note that this difference between the large and small angle of rotation problems occurred even though the difficulty of the different types of problems, as measured by the performance of the

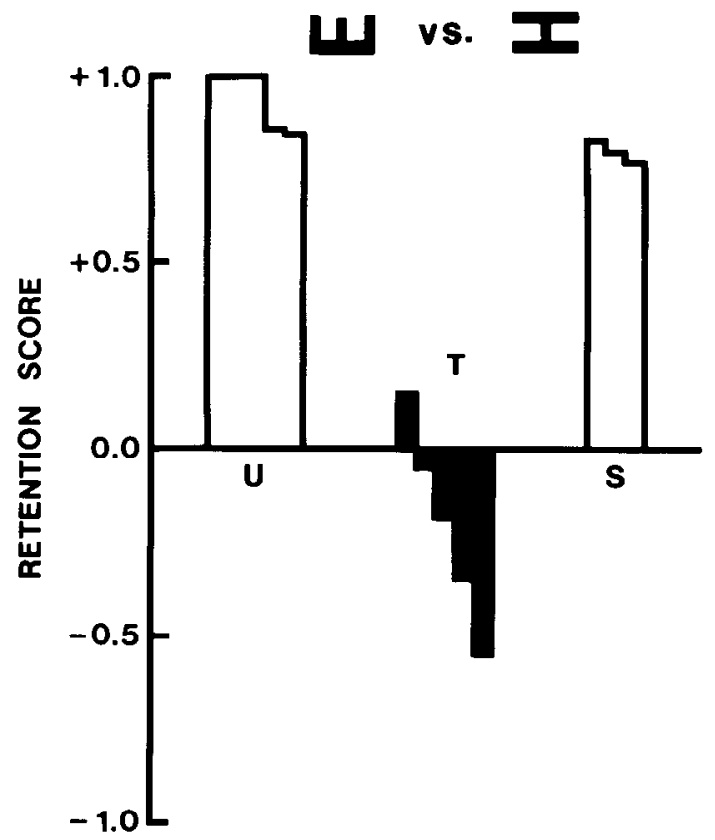

Figure 2. Retention scores (see the text) for the discrimination learned prior to operation for the unoperated $(U)$, inferior temporal $(T)$, and striate $(S)$ groups. Each bar within a group represents a single animal. Within the I'T group, from left to right, the individual bars represent animals IT- 1 to IT-5. The discriminanda are shown at the top in reverse contrast and were $3.2 \mathrm{~cm}$ long.

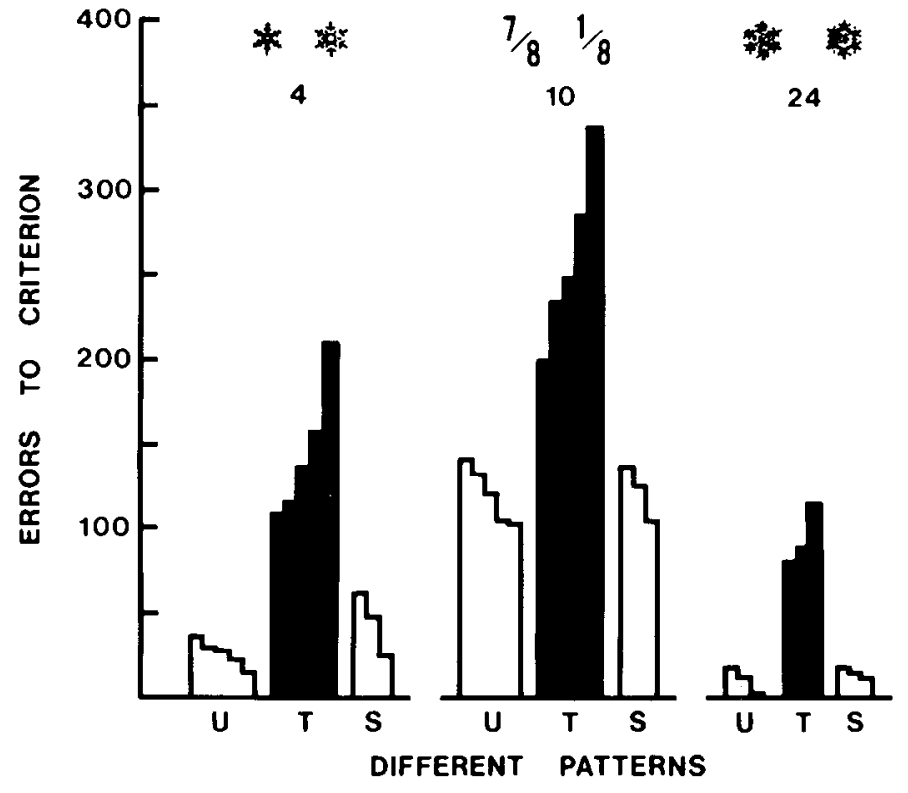

Figure 3. Errors madc prior to reaching criterion on the problems on which the discriminanda were different patterns. The problem number and the discriminanda are shown above each set of histograms. The discriminanda are shown in reverse contrast on the same scale as those in Figure 2 (see also legend to Fig. 2). In this and subsequent figures the bars for the individual animals are shown in the same order as in Figure 2.

unoperated animals, overlapped. Indeed the two most difficult problems (problems 2 and 6) were a $180^{\circ}$ and a $90^{\circ}$ rotation on both of which the IT group was unimpaired.

Different objects (Fig. 5). As had been the case with the different pattern problems, on all three problems in which the discriminanda were different three-dimensional objects, the IT group was severely and significantly impaired relative to both control groups $(U=0, p=0.05)$, whereas the control groups did not differ from each other $(U \geq 4)$.

Rotated objects (Fig. 5). On all six tasks on which the discriminanda were identical objects differing in orientation by $120^{\circ}$ (problems 14, 17, and 19) or $60^{\circ}$ (problems 15, 18, and 21), there were no significant or even suggestive differences among the group ( $U \geq 2$ ). By contrast, on the three problems in which the objects differed in orientation by $30^{\circ}$ (problems 16,20 , and 22 ), the IT group was severely impaired relative to the other groups $(U=0, p=0.05)$ which did not differ from each other $(U \geq 3)$.

Rotated line segments (Fig. 6). On the discrimination of horizontal and vertical lines (problem 1), the IT group made more errors (median $=84$ ) than did either the $U$ or the $\mathrm{S}$ groups (median $=58$ and 78 , respeclively), but the differences among the groups were not statistically significant. However, on both of the oblique line discriminations (problems 9 and 28) the IT group made many more errors than did the other two groups $(U=0, p=0.05)$ which did not differ from each other $(U \geq 2)$.

\section{Discussion}

In a previous study (Gross, 1978), we showed that monkeys with IT lesions were, paradoxically, not impaired in learning to discriminate two identical patterns differing in orientation by 90 or $180^{\circ}$, although, as expected, they were severely impaired in discriminating two different patterns (Gross, 1978). In the present study we replicated and extended these findings in several ways. First, we confirmed the previous results. The animals with IT lesions were not impaired on any of the seven discrimination tasks involving a 180 or $90^{\circ}$ difference in the 

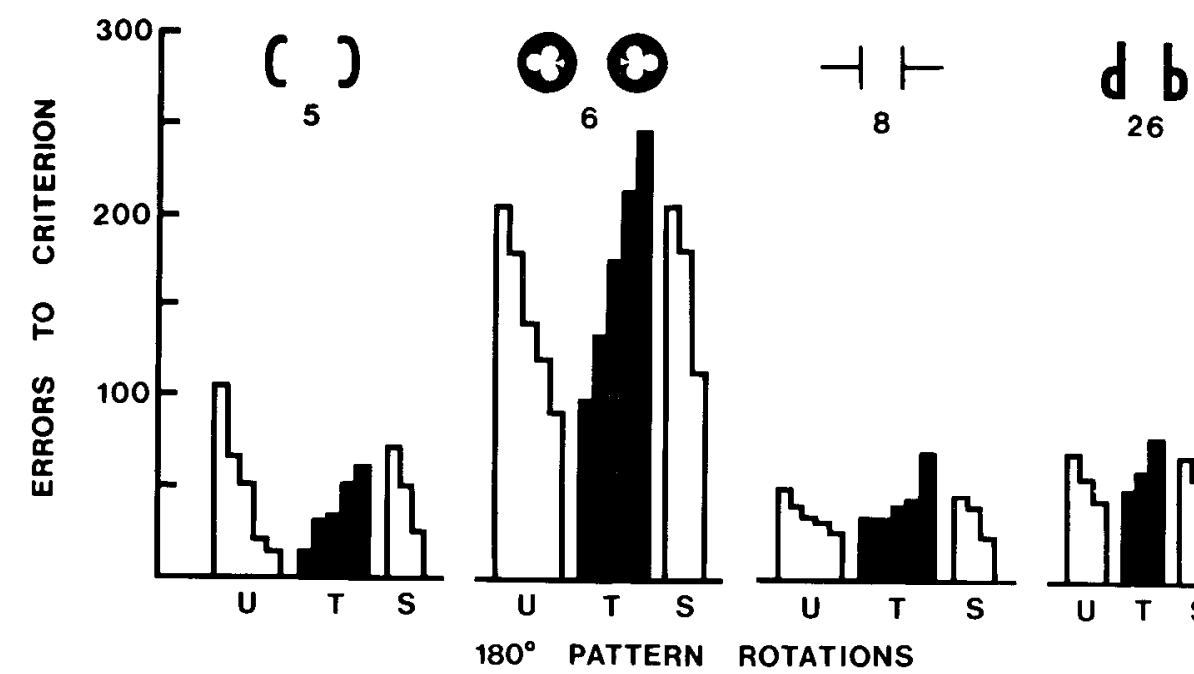

26
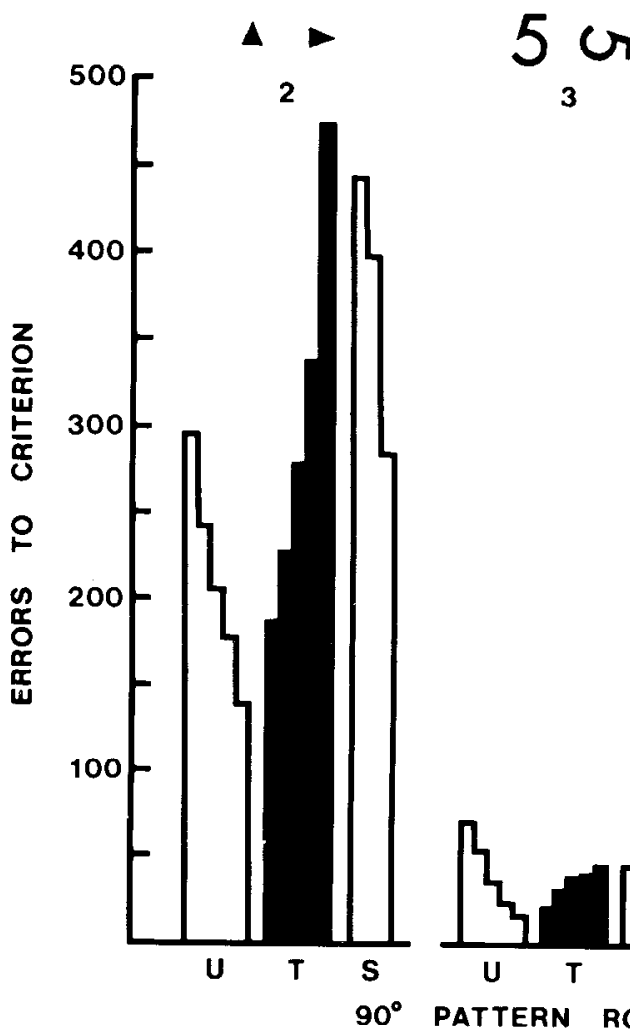

3
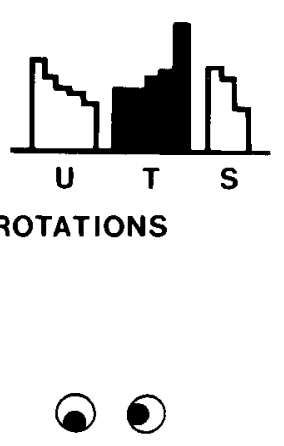

ROTATIONS

Figure 4. Errors made on the problems on which the discriminanda were identical patterns in different orientations. See also the legend to Figure 2.
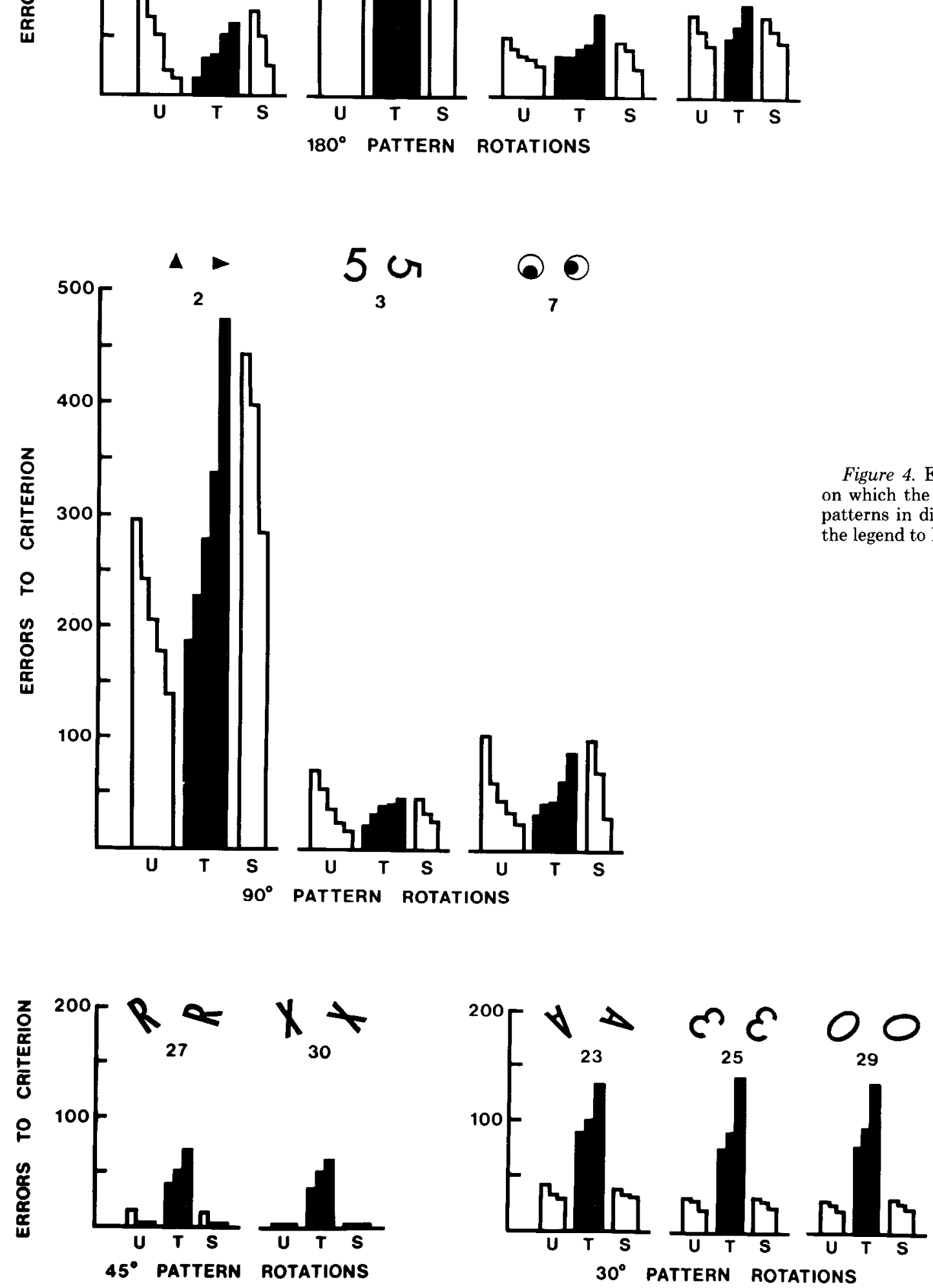

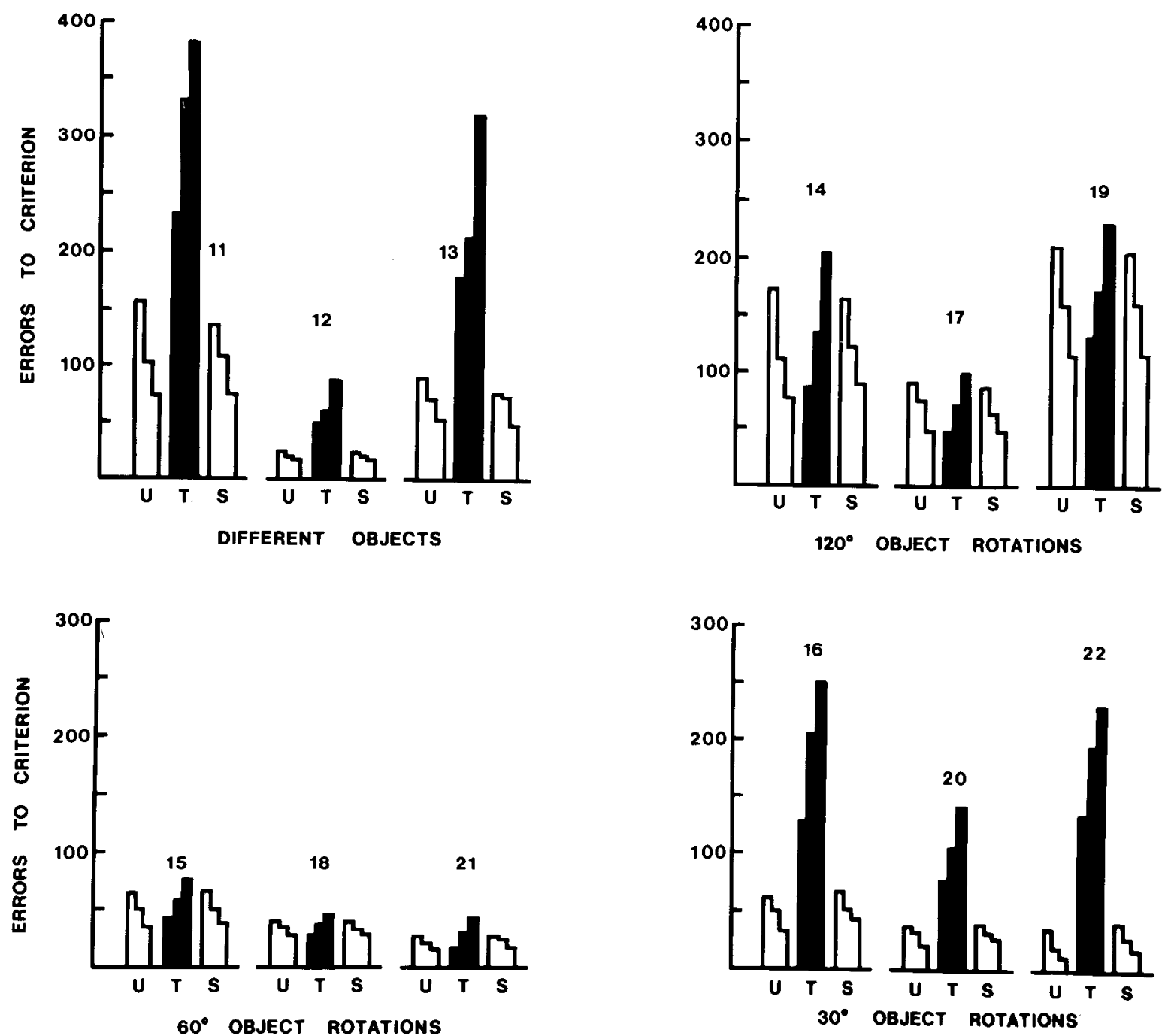

Figure 5. Errors made on the problems in which the discriminanda were three-dimensional objects. See also the legend to Figure 2.

orientation of identical patterns, although they were very deficient on all four discriminations involving different patterns. This result was not an artifact of problem difficulty because some of the rotation problems were actually much more difficult for the control animals than were some of the "different" problems. Second, we extended the previous finding of the absence of the IT deficit on rotated two-dimensional patterns to the case of rotated three-dimensional objects. The animals with IT lesions did not differ from controls on any of six problems in which the stimuli were identical objects rotated 120 or $60^{\circ}$, although they were severely impaired on all three problems involving different objects. Again, the difficulty of the rotated and different problems overlapped for the control animals. Third, we found that the absence of the IT deficit on rotated discriminanda requires at least a certain amount of rotation. The animals with IT lesions were severely impaired in all 7 cases in which the discriminanda differed by $45^{\circ}$ (patterns) or $30^{\circ}$ (patterns and objects), but not in any of the 13 cases in which the discriminanda differed by $60^{\circ}$ or more.

In the previous study (Gross, 1978) we had found one con- dition in which the animals with IT lesions had been impaired in discriminating stimuli differing only in orientation, namely, when the stimuli were line segments rather than patterns. In the current study, the IT animals were severely impaired on two of three rotated line segment problems.

In summary, there is a class of stimuli the discrimination of which is not impaired by IT lesions, namely, patterns and objects that differ from each other only in their orientation by $60^{\circ}$ or more. This sparing was not an artifact of problem difficulty, since many of these problems were as difficult for the normal animals as were the problems in which the animals with IT lesions were impaired. This sparing did not occur because the IT deficit had declined with time or training since the difference between rotated and different problems remained constant across the 18 months of postoperative training on the 30 problems.

Why was the IT deficit eliminated by the use of rotated but otherwise identical patterns as discriminanda, and why did the angular difference have to be $60^{\circ}$ or more? As previously suggested (Gross, 1978), the control animals may have initially 


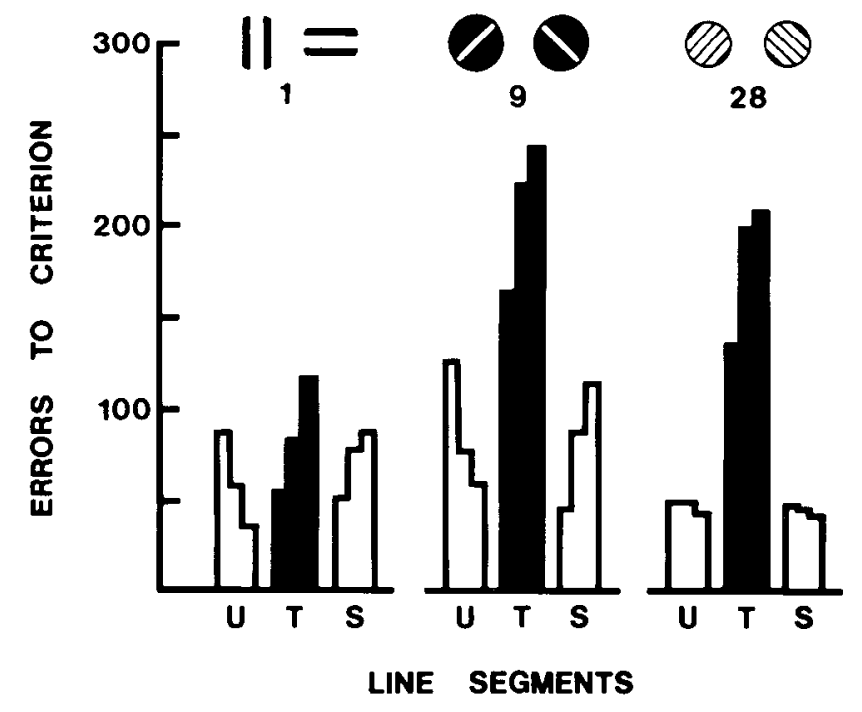

Figure 6. Frrors made on the problems in which the discriminanda were the illustrated line segments. See also the legend to Figure 2.

viewed the rotated patterns as equivalent to each other whereas the animals with IT lesions did not. That is, perhaps the control animals showed more "shape constancy," or "perceptual equivalence across rotation" or were more attentive to the whole figure rather than just a detail. If so, this would have made the discrimination relatively more difficult for the control animals and thereby would have reduced the difference between the control and IT groups. However, when the orientation difference between the stimuli was $45^{\circ}$ or less, both groups may have initially viewed the two stimuli as equivalent and the animals with IT lesions therefore lost their "advantage." According to this hypothesis, it should be possible to show a specific deficit in shape constancy after IT lesions. That is, if monkeys with IT lesions are trained to respond to a specific shape, they should show less transfer or stimulus equivalence when the shape is presented in a different orientation and perhaps a different size or contrast as well. (However, in a study subsequent to this one that involved some of the same animals, we failed to find support for a "shape constancy" deficit after IT lesions (Holmes and Gross, 1984).)

How can this hypothesis, that a deficit on shape constancy gives the animals with IT lesions a relative advantage in discriminating stimuli markedly different in orientation, explain the IT deficit when the rotated stimuli are line segments? Perhaps the nervous system treats line segments as literally one dimensional, that is, as shapeless and not subject to whatever mechanisms underlic shape constancy. The possibility that the IT cortex may process patterns and objects in a different fashion from line segments is consistent with the finding that single IT neurons almost invariably respond better to shapes and objects than to line segments (Schwartz et al., 1983; Desimone et al., 1984).

There is another possible explanation for the absence of a deficit after IT lesions on discrimination of patterns and objects differing only in orientation by $60^{\circ}$ or more. Perhaps normal monkeys solve such problems by utilizing postural or environmental cues. That is, normal animals may learn to perceive such stimuli as being "in-line" or "out of line" with the orientation of the body or some feature inside the test apparatus. Since monkeys with IT lesions are unimpaired on positional or landmark discrimination (Pohl, 1973), they may have solved the large orientation difference problems, but not the small ones as posilional or landmark discriminations. There is good evidence that the neural mechanisms underlying pattern and positional discrimination are different (Ungerleider and Mishkin, 1982). Not only do IT lesions impair pattern discrimination and not positional discrimination, but the opposite is true of posterior parietal lesions.

This hypothesis (i.e., that the sparing of impairment on discrimination of stimuli differing in orientation is because such problems are solved with a positional discrimination mechanism and not a pattern discrimination mechanism, and that the IT cortex is only needed for the latter) fails to explain the IT deficit on the rotated line segment problems. Such problems should be solvable with a positional mechanism. As noted above, the processing of line segment appears to be different from that of objects and patterns.

Since the early 1950 's, it has been clear that IT lesions impair visual discrimination (e.g., Mishkin and Pribram, 1954), but essentially nothing was known about what type of visual discrimination except the harder the task for normal animals, the greater the IT deficit. This and our previous study (Gross, 1978) provide the first specification of the visual stimuli that require the IT cortex for their discrimination. We have shown that the IT cortex is required for discriminating different visual patterns, not the same patterns differing markedly in orientation. The results also add further support to the indication from electrophysiological studies of the IT cortex that the processing of complex patterns by the IT cortex is different from that of line segments (Gross et al., 1972; Desimone et al., 1984).

\section{References}

Cowey, A., and C. G. Gross (1970) Effects of foveal prestriate and inferotemporal lesions on visual discriminations by rhesus monkeys. Exp. Brain Res. 11: 123-144.

Dean, P. (1976) The effects of inferotemporal lesions on the behavior of monkeys. Psychol. Bull. 83: 41-71.

Desimone, R., 'T. Albright, C. G. Gross, and C. Bruce (1984) Stimulusselective properties of inferior temporal neurons in the macaque. J. Neurosci. 4: 2051-2062.

Gross, C. G. (1973) Inferotemporal cortex and vision. In Progress in Physiological Psychology, E. Stellar and J. M. Spraque, eds., Vol. 5, pp. 77-123, Academic Press, Inc., New York.

Gross, C. G. (1978) Inferior temporal lesions do not impair discrimination of rotated patterns in monkeys. J. Comp. Physiol. Psychol. 92: 10951109 .

Gross, C. G., C. E. Rocha-Miranda, and D. B. Bender (1972) Visual properties of neurons in inferotemporal cortex of the macaque. J. Neurophysiol. 35: 96-111.

Holmes, E. J., and C. G. Gross (1984) Stimulus equivalence after inferior temporal lesions in monkeys. Behav. Neurosci. 98: 898-901.

Mishkin, M., and K. H. Pribram (1954) Visual discrimination performance following partial ablations of the temporal lobe. I. Ventral vs. lateral. J. Comp. Physiol. Psychol. 47: 14-20.

Pohl, W. (1973) Dissociation of spatial discrimination deficits following frontal and parietal lesions in monkeys. J. Comp. Physiol. Psychol. 82: 227-239.

Schwartz, E. L., R. Desimone, T. D. Albright, and C. G. Gross (1983) Shape recognition and inferior temporal neurons. Proc. Natl. Acad. Sci. U. S. A. 80: 5776-5778.

Ungerleider, L. G., and M. Mishkin (1982) Two cortical visual systems. In Analysis of Visual Behavior, D. J. Ingle, M. A. Goodale, and R. J. W. Mansfield, eds., pp. 459-586, MIT Press, Cambridge, MA.

von Bonin, G., and P. Bailey (1947) The Neocortex of Macaca Mulatta, University of Illinois Press, Urbana, IL. 